\title{
INCORPORATION OF DIFFRACTION EFFECTS IN SIMULATIONS OF ULTRASONIC SYSTEMS USING PSPICE MODELS
}

\author{
Jonny Johansson and Per-Erik Martinsson \\ Luleå University of Technology \\ 97187 Luleå, Sweden
}

\begin{abstract}
The use of PSpice models for piezoelectric devices and ultrasonic transmission media is of major importance in the design of electronics for ultrasonic systems. Today, these models include viscoelastic loss but disregard loss due to diffraction, i.e. beam spreading. This paper presents a method to include diffraction loss in PSpice simulations of ultrasonic systems. The conductive loss in the transmission line, that models the propagation media of the ultrasound pulse, is used to model the loss due to diffraction. Parameter variations for the piezoelectric device can affect the result greatly. Thus, a sensitivity analysis for the simulation model is presented.

Measurements and simulations have been performed using a pulse echo system in water. Maximum distance to the reflector was $200 \mathrm{~mm}$. The piezoelectric devices used were PZ-27 crystals with diameters $6 \mathrm{~mm}$ and $12 \mathrm{~mm}$, with a center frequency of $4 \mathrm{MHz}$. Results show that the simulated amplitude of the echo follows measured values well in both near and far fields, with an offset of about $10 \%$.
\end{abstract}

\section{INTRODUCTION}

The use of simulation tools intended for electronic circuits (e.g. SPICE, PSpice) is well established in the design of ultrasonic systems. Equivalent circuits for piezoelectric elements using passive components were initially developed in [1 - 3]. Leach [4] used controlled sources to avoid negative capacitance and frequency dependant transformers. Püttmer et al [5] included losses in this model to model low-Q thickness mode transducers. Also, losses were introduced in the transmission media to give the attenuation of the propagating wave. Van Deventer et al [6] further investigated material properties governing viscoelastic loss and wave speed. However, none of the works hitherto presented has concerned simulations giving absolute amplitudes of received signals. In the design of electronics for an ultrasonic system absolute amplitudes are of importance, as they set required dynamics in the electronic system. If correct amplitudes can be achieved in system simulations, electronic designs can be built solely based on simulation results. One important factor when aiming for correct amplitude is the loss introduced in a system due to diffraction, i.e. beam spreading. The paper at hand presents a method to include diffraction loss in a pulse echo simulation, in order to achieve absolute amplitude correctness. Material parameters used have a large influence on received echo amplitudes. Therefore, a sensitivity analysis for the simulation model is presented.

\section{METHOD}

\section{Modeling the diffraction effect}

When an ultrasonic system is simulated with PSpice, electrical transmission lines are used to model the propagation medium $[5,6]$. Under the assumption of low-loss conditions $(R<<\omega L, G<<\omega C)$, the attenuation constant $\alpha$ $(\mathrm{Np} / \mathrm{m})$ for an electrical transmission line can be written [6]:

$$
\alpha=\frac{1}{2} \sqrt{L C}\left(\frac{R}{L}\right)+\frac{1}{2} \sqrt{L C}\left(\frac{G}{C}\right)
$$

Here $R(\Omega / \mathrm{m})$ is resistive loss, $G(1 / \Omega \mathrm{m})$ is conductive loss, $C(\mathrm{~F} / \mathrm{m})$ is capacitance, and $L(\mathrm{H} / \mathrm{m})$ is inductance per unit length. This can be rewritten

$$
\alpha=\frac{1}{2} \frac{R}{Z_{0}}+\frac{1}{2} G Z_{0}
$$

where $Z_{0}=\sqrt{L / C}$ is the characteristic impedance of the transmission line. A propagating sound wave is modeled as a forward traveling voltage wave in the transmission line. The amplitude of this voltage wave can be expressed as [7]:

$$
|V(x)|=\left|V_{0}\right| e^{-\alpha x},
$$

where $V_{0}$ is the voltage amplitude at $x=0$. Using (2) we get

$$
|V(x)|=\left|V_{0}\right| e^{-\left(R / 2 Z_{0}\right) x} e^{-\left(G Z_{0} / 2\right) x} .
$$

Previous works on simulation of ultrasound propagation 


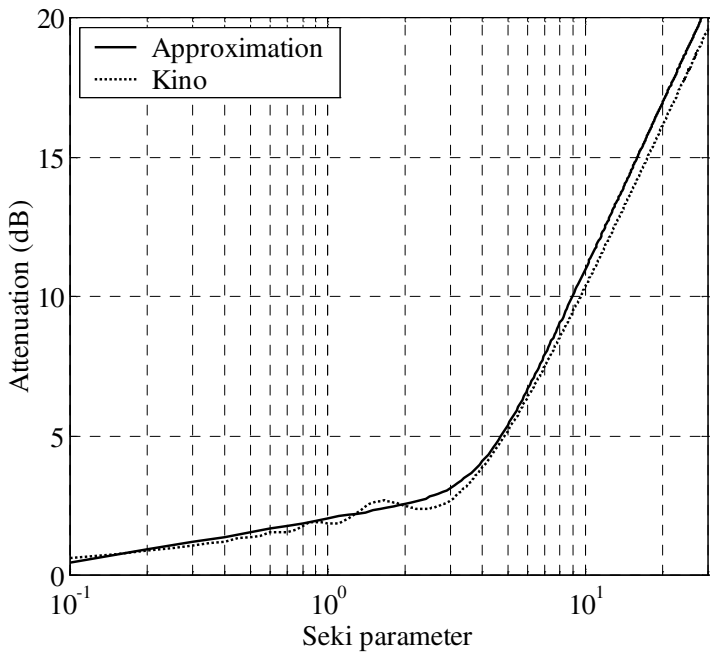

Figure 1: Diffraction loss after Kino vs. Approximation

using electrical transmission lines have used $R$ to model viscous losses while setting $G$ to $0[5,6]$. Here, by using $G$ $\neq 0$ we can add an attenuation term non-dependant of the attenuation caused by $R$. This can be used to model the attenuation due to diffraction as

$$
A_{d i f f}=\frac{|V(x)|}{\left|V_{0}\right| e^{-\left(R / 2 Z_{0}\right) x}}=e^{-\left(G Z_{0} / 2\right) x} .
$$

Solving for $\mathrm{G}$ gives

$$
G=\frac{2}{Z_{0} x} \ln \left(A_{\text {diff }}\right),
$$

which can be used as a parameter in PSpice once the required diffraction loss is known.

Given two identical transducers with radius $a$ spaced a distance $x$ apart, the diffraction loss when transmitting from one to another is given by Kino [8] as

$$
A_{\text {loss }}=\left|2 \int_{0}^{\infty} \frac{J_{1}^{2}(y)}{y} e^{j y^{2} S / 4 \pi} d y\right| .
$$

Here

$$
S=x \lambda / a^{2}
$$

is the Seki parameter [9], and $\lambda$ is the wavelength of the transmitted ultrasound pulse. The integrand is
Table 1: Simulation data for PZ-27.

\begin{tabular}{lll}
\hline Parameter & Notation & Value \\
\hline Radius & $a(\mathrm{~mm})$ & $3 / 6$ \\
Density & $\rho_{P Z}\left(\mathrm{~kg} / \mathrm{m}^{3}\right)$ & 7700 \\
Thickness & len $(\mathrm{m})$ & $487 \cdot 10^{-6}$ \\
Permittivity & $\mathcal{E}^{S}(\mathrm{As} / \mathrm{Vm})$ & $8.09 \cdot 10^{-9}$ \\
Piezoel. stress const. & $e^{33}\left(\mathrm{C} / \mathrm{m}^{2}\right)$ & 16 \\
Q factor & $Q$ & 74 \\
Conductivity & $G(1 / \Omega)$ & 0 \\
Elastic constant & $C^{E}(\mathrm{~Pa})$ & $1.13 \cdot 10^{11}$ \\
\hline
\end{tabular}

Table 2: Simulation data for PMMA, water and steel.

\begin{tabular}{lll}
\hline Parameter & Notation & Value \\
\hline Density of water & $\rho_{w a t}\left(\mathrm{~kg} / \mathrm{m}^{3}\right)$ & 998.2 \\
Speed of sound, water & $c_{\text {wat }}(\mathrm{m} / \mathrm{s})$ & 1478 \\
Viscous loss factor & $\alpha_{V}(\mathrm{~Np} / \mathrm{m})$ & 0.13 \\
Density of steel & $\rho_{s t}\left(\mathrm{~kg} / \mathrm{m}^{3}\right)$ & 8000 \\
Speed of sound, steel & $c_{s t}(\mathrm{~m} / \mathrm{s})$ & 5900 \\
Density of PMMA & $\rho_{P M M A}\left(\mathrm{~kg} / \mathrm{m}^{3}\right)$ & 1190 \\
Speed of sound, PMMA & $c_{P M M A}(\mathrm{~m} / \mathrm{s})$ & 2775 \\
\hline
\end{tabular}

$$
y=k_{r} a
$$

in which $k_{r}$ is the radial propagation constant. For a pulse echo system with a perfect plane reflector, $x$ in (8) is replaced with $2 x$, where $x$ is the distance to the reflector [8]. $A_{\text {loss }}$ as given by (7) is plotted for $1 \leq S \leq 30$ in figure 1 .

As equation (7) must be solved numerically, the use of it as a parameter in PSpice simulations is not straightforward. From figure 1 it is seen that the behavior of (7) can be approximated with two straight lines in a log-log scale. Thus, an analogy with electrical filters was used to create an approximation of the diffraction loss. Two "filter" sections were used, one to create a straight line for $S \leq 4$,

$$
A_{1}=\frac{1}{1.05 \cdot(S / 0.1)^{0.08}},
$$

and one to increase the slope for $S \geq 4$ : 


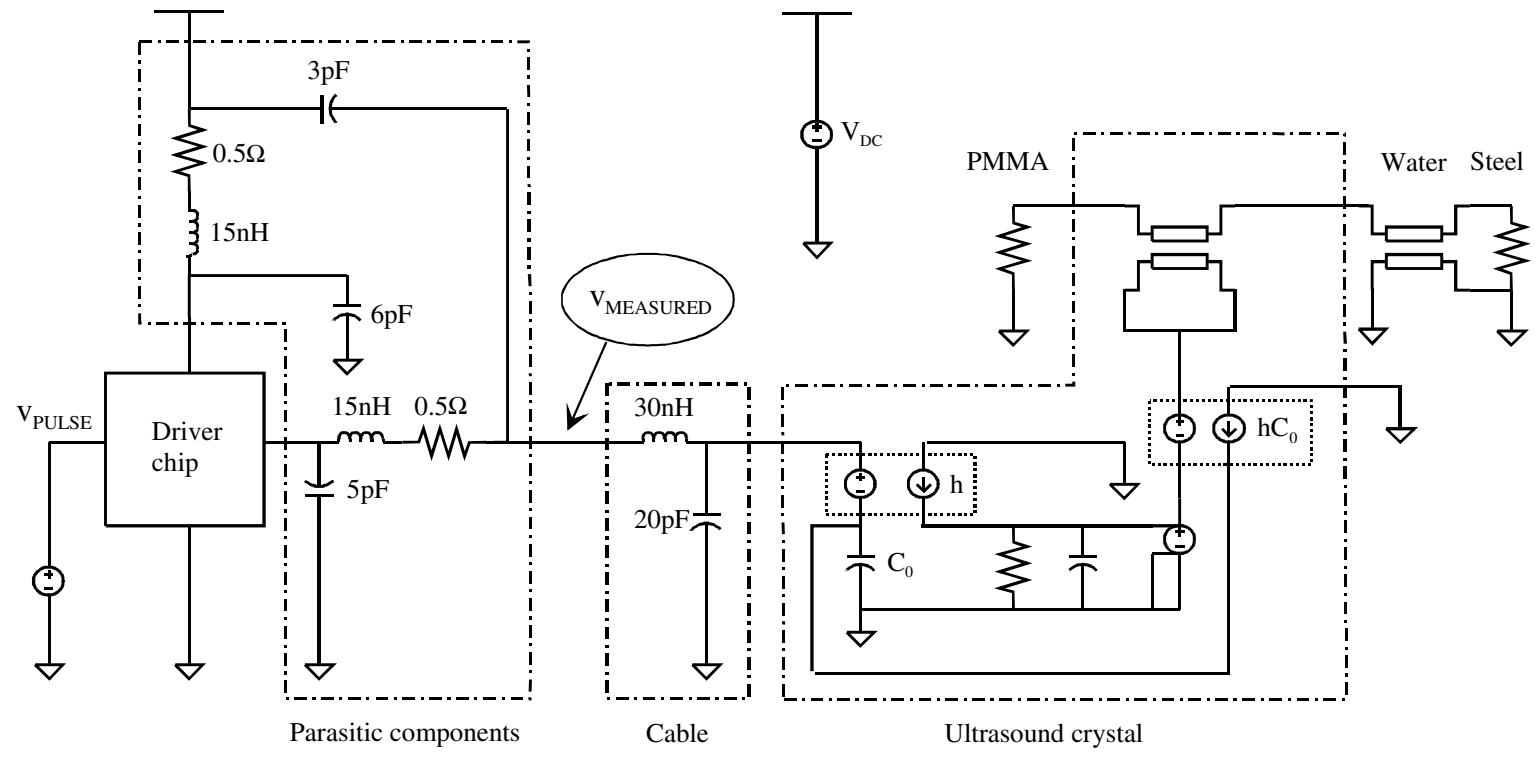

Figure 2: Schematic of the simulation.

$$
A_{2}=\frac{1}{\left[(1+S / 4)^{5}\right]^{0.184}} .
$$

The total attenuation is given by

$$
A_{\text {tot }}=A_{1} \cdot A_{2} .
$$

The exponents for the denominators in (10) and (11) are chosen to give $A_{t o t}$ a slope of $1 / S$ in the far field for $S>4$, following the Fraunhofer approximation [8]. The order of the "filter section" in (11) is chosen to match the roll-off to the curve given by (7). $A_{t o t}$ is plotted for $1 \leq S \leq 30$ in figure 1. It is seen here that the small variations in attenuation for $S \leq 2$ are excluded in the approximation. If these were to be included, the model could easily be extended using more "filter sections" containing higher order terms of $S$. In this paper, they have been omitted for clarity. Using (6) and (12) and setting

$$
A_{\text {diff }}=A_{\text {tot }}
$$

now gives a complete set of input parameters to model diffraction loss in a PSpice simulation. The method described above is applicable only if the diffraction occurs mainly in one medium, i.e. if other parts of the propagation path are thin enough that diffraction in these can be considered negligible.

\section{Simulation setup}

The schematic used for simulation is shown in figure 2. Simulations were performed using the Cadence Spectre simulation engine. Spectre has the possibility to run netlists created for PSpice, enabling the use of models previously developed for PSpice. The model used for the simulation of the piezoceramic disc was initially presented by Leach [4], further developed by Püttmer [5] and van Deventer [6]. Also the medium through which the ultrasonic pulse is transmitted is modeled as a transmission line, with data calculated using methods from [6]. Simulation of the electronics is made using transistor models from the semiconductor manufacturer. Estimated parasitic components are included in the simulation schematic. The temperature for the simulations is $20^{\circ} \mathrm{C}$. Parameter data for PZ-27 used in the simulation are shown in table 1 [10]. From [8] we get the equation for stiffened elastic constant

$$
C^{D}=C^{E}\left(1+\frac{\left(e^{33}\right)^{2}}{\varepsilon^{S} C^{E}}\right)
$$

Van Deventer [6] developed expressions for the loss resistance in the model

$$
R_{P Z}=\frac{2 \pi^{2} f a^{2} \rho_{P Z}}{Q},
$$

the characteristic impedance 


$$
Z_{0 P Z}=\rho_{P Z} \pi a^{2} c_{P Z}
$$

and the transmitting constant

$$
h=\frac{e^{33}}{\varepsilon^{S}}
$$

Further, the speed of sound in PZ-27 is [8]:

$$
c_{P Z}=\sqrt{\left(\frac{C^{D}}{\rho_{P Z}}\right)} .
$$

Parameter data used for PMMA [6], water [6] and steel [11] are shown in table 2. From [6] we get formulae for the calculation of lumped backing and reflector impedances for steel and PMMA as

$$
Z=\rho c \pi a^{2}
$$

Viscoelastic loss in the transmission line that models water is given by:

$$
R_{w a t}=2 \rho_{w a t} c_{w a t} \pi a^{2} \alpha_{v}
$$

\section{Experimental setup}

The system investigated is a pulse echo system, where the transducer is immersed in water as shown in figure 3. An ultrasonic pulse is sent towards a steel reflector and is received with the sending device. The piezoceramic element used is a PZ-27 disc manufactured by Ferroperm, Denmark. The disc is mounted with cyanoacrylate glue on a carrier made of plexiglas (PMMA) to form a transducer unit. The plexiglas is used as backing and is shaped to reduce any echo returned to the rear side of the disc. The front side of the disc together with electrical connections is covered with one layer of PC-52 protective lacquer to allow immersion in water. The thin layer of lacquer does not significantly affect the measurements, and can thus be ignored in the simulations [12]. The transducer unit is mounted to a co-ordinate table, allowing both translational and rotational motion. The transducer was adjusted to be parallel to the steel reflector by observing when the returned echo amplitude was maximized.

The electronics used is a custom-made driver chip [13]. The chip is a push-pull driver design, generating a square wave excitation pulse. The driver is controlled by a signal generator with the pulse width set to half the crystal oscillating period time, to give maximum returned echo amplitude [14]. The driver chip is connected to the piezoelectric crystal with a $0.2 \mathrm{~m}$ long coaxial cable. Measurements of

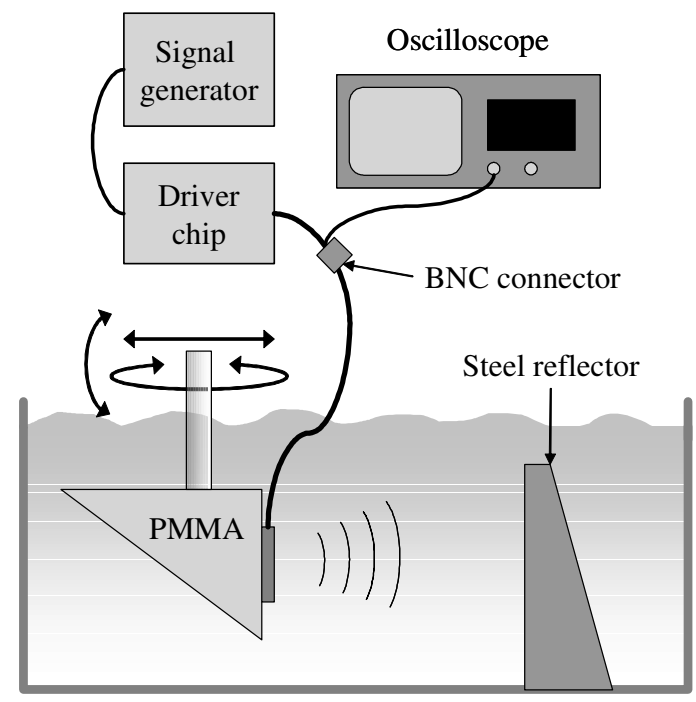

Figure 3: Measurement setup.

received echo signals are made on the chip side of the cable using a Tektronix TDS724 oscilloscope. The oscilloscope has an accuracy of $1 \%$ and a resolution of 8 bits.

\section{MEASUREMENTS}

Measurements were performed for two different transducers of diameters $6 \mathrm{~mm}$ and $12 \mathrm{~mm}$. The thickness of the discs is $0.5 \mathrm{~mm}$ giving a resonance frequency of about 4 MHz. Results from the measurements are shown in figures 4 and 5. The far field limit where the sound pulse starts a more rapid divergence is clearly seen for the smaller transducer (fig. 4) at a reflector distance of $30 \mathrm{~mm}$. The far field limit for the larger transducer (fig. 5) is about $120 \mathrm{~mm}$. Water can be considered to be a good carrier of ultrasonic waves, with attenuation due to viscoelastic losses of about $1.1 \mathrm{~dB} / \mathrm{m}$ [6]. This verifies that the measured attenuation profiles presented in figures 4 and 5 are indeed produced by diffraction effects.

\section{Simulations}

Initial simulations were made with the diameter of the piezoelectric disc set to $6 \mathrm{~mm}$ and $12 \mathrm{~mm}$. Results from these simulations are shown in figure 4 and 5 respectively, together with measured results. From the outcome of these simulations, it was clear that the diffraction loss was underestimated as the whole curve was shifted towards too high values along the $\mathrm{x}$-axis. In the simulation model, the parameter $S$ as defined in (8) is decisive for the behavior of the curve. $S$ in turn is highly dependant on the transducer radius, $a$. If $a$ is decreased, the $S$ values will increase and the curves will be shifted towards smaller values along the 


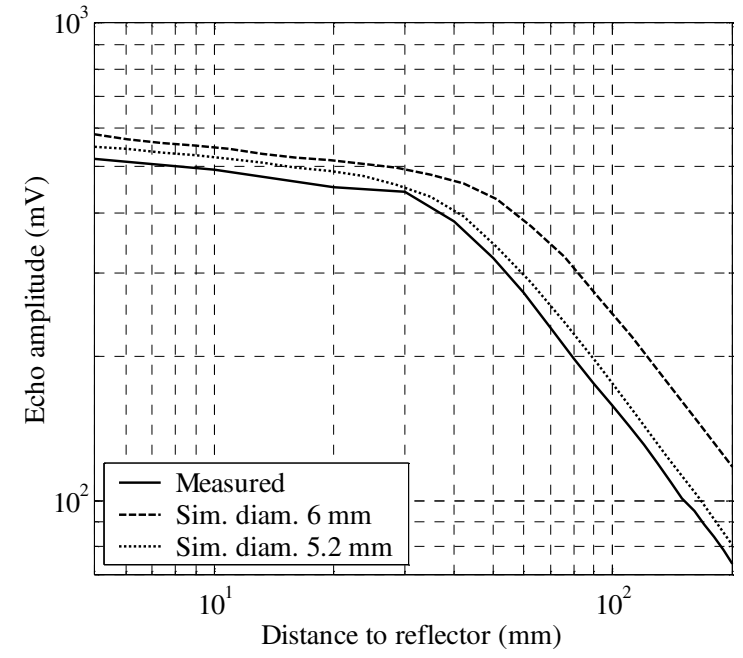

Figure 4: Simulated vs. measured echo amplitudes for $\varnothing=6 \mathrm{~mm}$.

$\mathrm{x}$-axis. Thus, the effective diameter in the model was decreased in order to find a better match to the measurements. From the initial results it was seen that the curve should be shifted $25-30 \%$ to the left. This corresponds to a decrease in diameter of $13-16 \%$. It was found that decreasing the diameter $13 \%$ gave a correct limit between nearand far fields, leaving an offset of less than $10 \%$. Simulated results with diameters set to $5.2 \mathrm{~mm}$ and $10.4 \mathrm{~mm}$ are plotted in figures 4 and 5 respectively. The reduction in diameter agrees well with experimental data by Almqvist [15], who reports an effective diameter being $14 \%$ smaller than the physical size of a transducer.

\section{DISCUSSION}

The reduction in diameter is applied throughout the model of the piezoelectric crystal, both in mechanical and electrical parts. Whether this is the correct approach or not is a matter for future research. Three possibilities can be seen. The first is the taken approach. This makes sense for the mechanical side, as reported by Almqvist [15]. However, it also affects the electrical side, reducing the value of the static capacitance $C_{0}$, affecting the energy required to excite the transducer. One way to keep $C_{0}$ at nominal value would be to apply the reduction on the mechanical side only. With this approach a decision has to be taken regarding the dependent source with value $h C_{0}$, as shown in figure 2. Should this source rely on a capacitance value calculated after nominal or reduced diameter in this case? The third approach is to keep nominal diameters throughout the simulation model, and apply the reduced value only in the

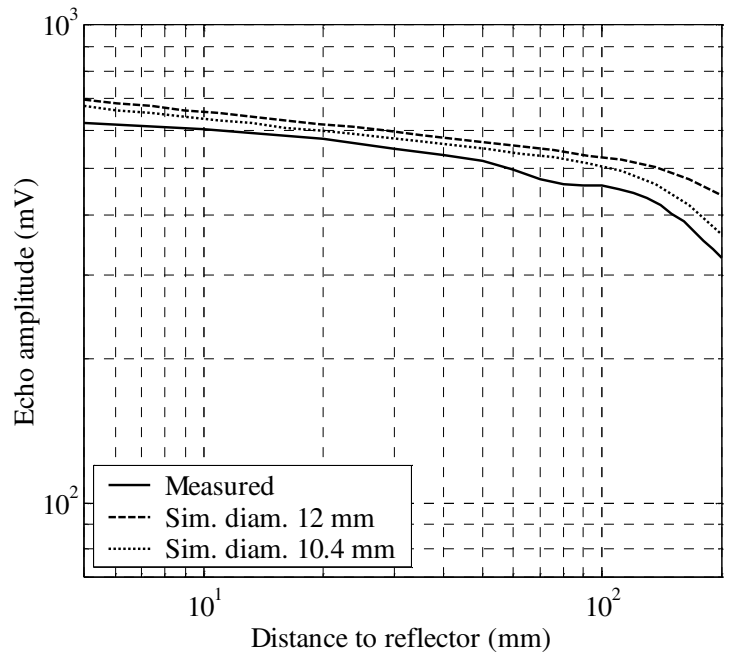

Figure 5: Simulated vs. measured echo amplitudes for $\emptyset=12 \mathrm{~mm}$.

calculation of the conductive loss $G$ in the transmission line.

As noted above, an offset of less than $10 \%$ persists when the attenuation curve has been shifted to achieve correct far field limit. This offset may well be due to tolerances in material parameters, which normally are given to $\pm 10 \%$. Variations in material parameters will also affect the frequency and thereby the wavelength. This will change the Seki parameter $S$ and shift the diffraction curve along the $\mathrm{x}$-axis as discussed above.

To investigate the influence of parameter variations, a simulated sensitivity analysis was performed. Two distances were chosen, $5 \mathrm{~mm}$ (near field), and $200 \mathrm{~mm}$ (far field). The crystal diameter used was $5.2 \mathrm{~mm}$. Parameter values were increased one by one with $10 \%$, and the resulting change in received echo amplitude was recorded. Although this simple analysis does not include correlation effects, it does give an indication of the relative influence of the parameters [16]. Results from the simulations are shown in table 3. It is seen that variations in material parameters can have major effect on the received echo amplitude.

Uncertainties exist also in the simulation of the electronics. Variations in performance occur between produced batches of silicon chips. The manufacturer deals with this by giving the circuit designer models of the process in so called "corners" [17]. Three corners given may be typicalmean, fast, and slow. These will give simulations with typical, fast, and slow performance of the components respectively. Furthermore, the temperature dependence of the circuit performance can be high. The simulations presented in this paper are run with performance set to typical-mean, 
Table 3: Results of sensitivity analysis

\begin{tabular}{lcc}
\hline & \multicolumn{2}{c}{ Change in Echo Amplitude (\%) } \\
Parameter & Near Field & Far Field \\
\hline$\varepsilon^{S}$ & -5.8 & -5.9 \\
$e^{33}$ & 14.4 & 15.6 \\
$Q$ & 1.2 & 1.5 \\
$\rho_{\text {PZ27 }}$ & -2.8 & -5.1 \\
$C^{E}$ & -8.7 & -5.5 \\
$Z_{\text {PMMA }}$ & -5.7 & -5.0 \\
$Z_{\text {WATER }}$ & 6.2 & 6.5 \\
$Z_{\text {STEEL }}$ & 0.2 & 0.7 \\
\hline
\end{tabular}

and temperature set to $20^{\circ} \mathrm{C}$. To get a complete view of the system behavior under real operating conditions, simulations should be run in various corner settings and temperatures as recommended by the manufacturer of the chip. Another possibility is to use Monte Carlo simulations to randomly vary a number of parameters in the electronics.

\section{CONCLUSIONS}

This paper has presented a method to include diffraction loss in PSpice simulations of ultrasonic systems. Conductive loss in the transmission line modeling the propagation media of the ultrasound pulse is used to model the diffraction loss. The method can be used either for systems employing separate transmitter/receivers of equal size, or in a pulse echo system. The method is limited to systems where the diffraction loss occurs mainly in one part of the transmission path. For a pulse echo system in water, good agreement is shown between simulated and measured results. Simulated echo amplitudes are within $10 \%$ of measured data in both near and far fields. To achieve these results, a reduction with $13 \%$ is made in the effective radius of the transducer. The method used to achieve the reduced radius is a subject for further research.

\section{REFERENCES}

[1] W.P. Mason, Electromechanical Transducers and Wave Filters, New York: Van Nostrand, 1942.

[2] M. Redwood, "Transient performance of a piezoelectric transducer", J. Acoust. Soc. Amer., vol. 33, pp. 527-536, Apr. 1961.

[3] R. Krimholtz, D.A. Leedom, and G.L. Matthei, "New equivalent circuits for elementary piezoelectric transducers", Electron. Lett., vol. 6, pp. 398399, June 1970.
[4] W.M. Leach, "Controlled-Source Analogous Circuits and SPICE Models for Piezoelectric Transducers", IEEE Trans. Ultrason., Ferroelect., Freq. Contr., Vol. 41, No. 1, pp. 60-66, Jan. 1994.

[5] A. Püttmer, P. Hauptmann, R. Lucklum, O. Krause, and B. Henning, "SPICE Model for Lossy Piezoceramic Transducers", IEEE Trans. Ultrason., Ferroelect., Freq. Contr., Vol. 44, No. 1, pp. 60-66, Jan. 1997

[6] J.A. van Deventer, T. Löfqvist, and J. Delsing, "PSpice simulation of ultrasonic systems", IEEE Trans. Ultrason., Ferroelect., Freq. Contr., Vol. 47, No. 4, pp. 1014-1024, July 2000.

[7] R. Ludwig, P. Bretchko, RF Circuit Design, Upper Saddle River, NJ: Prentice-Hall 2000.

[8] G.S. Kino, Acoustic Waves: Devices, Imaging and Analog Signal Processing. Englewoods Cliffs, NJ: Prentice-Hall, 1988.

[9] H. Seki, A. Granato, and R. Truell, "Diffraction Effects in the Ultrasonic Field of a Piston Source and Their Importance in the Accurate Measurement of Attenuation", The J. Acoust. Soc. Amer., Vol. 28, No 2, pp. 230-238, Mar. 1956.

[10] Homepage of Ferroperm A/S, Denmark: http://www.ferroperm.com/, Visited 2001-02-21.

[11] C. Nordling, J Österman, Physics Handbook, Lund, Studentlitteratur 1988.

[12] M. Willatzen, "Ultrasound Transducer Modeling Received Voltage Signals and the Use of HalfWavelength Window Layers with Acoustic Coupling Layers", IEEE Trans. Ultrason., Ferroelect., Freq. Contr., Vol. 46, No. 5, pp. 1164-1174, Sep. 1999.

[13] J. Johansson, "Optimization of a Piezoelectric Crystal Driver Stage using System Simulations", Proc. 2000 IEEE Ultrason. Symp., Vol. 2, pp 1049-1054, 2000.

[14] H.W. Persson, "Electric excitation of ultra-sound transducers for short pulse generation", Ultrasound in Med. \& Biol., Vol. 7, pp. 285-291, 1981.

[15] M. Almqvist, "High resolution light diffraction tomography: nearfield measurements of $10 \mathrm{MHz}$ continuous wave ultrasound", Ultrasonics, vol.37, no.5; p.343-53; June 1999.

[16] G.E.P. Box, W.G. Hunter, J.S. Hunter, Statistics for Experimenters: an introduction to design, data analysis, and model building, New York, Wiley \& Sons, 1978.

[17] D. Foty, MOSFET Modeling with SPICE, Upper Saddle River, N.J., Prentice Hall, 1997. 